\title{
Neuroendocrine Control of Larval Ecdysis Behavior in Drosophila: Complex Regulation by Partially Redundant Neuropeptides
}

\author{
Anthony C. Clark, ${ }^{\star}$ Marta L. del Campo, ${ }^{\star}$ and John Ewer \\ Cornell University, Entomology Department, Ithaca, New York 14853
}

To complete each molting cycle, insects display a stereotyped sequence of behaviors to shed the remains of the old cuticle. These behavioral routines, as well as other related physiological events, are critical for proper development and are under the control of several neuropeptides. Their correct deployment and concatenation depends on the complex actions and interactions among several peptide hormones: ecdysis triggering hormone (ETH), eclosion hormone (EH), and crustacean cardioactive peptide (CCAP). Numerous theories, some in conflict, have been proposed to define the functional hierarchies by which these regulatory factors operate. Here we use wild-type Drosophila and transgenic flies bearing targeted ablations of either EH or CCAP neurons, or ablations of both together, to reevaluate their roles. Consistent with findings in moths, our results suggest that EH and ETH affect the release of each other via a positive feedback, although ETH can also be released in the absence of EH. We show that EH and ETH both contribute to the air filling of the air ducts (trachea) of the next stage but that EH may play a primary role in this process. We present evidence that EH, whose actions have always been placed upstream of CCAP, may also regulate ecdysis independently of CCAP. Finally, we confirm that flies lacking EH neurons do not ecdyse prematurely when injected with ETH peptides. These findings are surprising and not easily explained by currently available hypotheses. We propose that important additional neuropeptides, and additional interactions between known regulators, contribute to the mechanisms underlying insect ecdysis behaviors.

Key words: eclosion hormone; ecdysis triggering hormone; CCAP; neurohormone; molt; neurosecretion; cGMP

\section{Introduction}

Neuropeptides are small signaling molecules that play important roles in the development, physiology, and behavior of both vertebrates and invertebrates. Research conducted over the past few decades has documented the enormous diversity of neuropeptides, their phylogenetically widespread occurrence, and the complex nature of their actions (Strand, 1999; Nässel, 2002). For example, a given neuropeptide can effect changes in central and peripheral targets, and different neuropeptides can act on the

Received Nov. 4, 2003; revised March 3, 2004; accepted March 17, 2004.

This work was supported by United States Department of Agriculture (USDA) National Research Initiative Competitive Grants Program Grant 2000-02829 and Cooperative State Research, Education, and Extension Service USDA Hatch Grant NYC-139407. We thank Ziren Zhou and Qasim Husain for help in analyzing videos of larval behavior and Ken Kemphues for the use of time-lapse video recorder. We are very grateful to Hans Jürgen Agricola for anti-CCAP, Michael Adams and Yooseong Park for the gifts of anti-ETH1 and synthetic DmETH1 and DmETH2, Andrea Novicki for synthetic MasETH, Klaude Weiss for anti-MM, and Jim Truman for anti-EH. We thank Sue McNabb and Jim Truman for providing EH-GAL4 transgenic flies, Hermann Steller for UAS-reaper flies, and the Bloomington Stock Center for UAS-LacZ flies. We thank Paul Taghert and Rob Jackson for helpful comments and discussions on this manuscript and Ralph Greenspan for insights into biological degeneracy.

${ }^{*}$ A.C.C. and M.L.d.C. contributed equally to this work

Correspondence should be addressed to John Ewer, Entomology Department, Cornell University, 5130 Comstock Hall, Ithaca, NY 14850. E-mail: je24@cornell.edu.

A. C. Clark's present address: Division of Newborn Medicine, Children's Hospital Boston, Enders 9, Boston, MA 02115.

M. L. del Campo's present address: Department of Biological Sciences, State University of New York, P.0. Box 6000 , Binghamton, NY 13902-6000.

DOI:10.1523/JNEUROSCI.4938-03.2004

Copyright $\odot 2004$ Society for Neuroscience $\quad$ 0270-6474/04/244283-10\$15.00/0 same target. The tractable nervous system of invertebrates has provided key insights into many aspects of neuropeptide biology. In particular, the analysis of insect ecdysis behavior has greatly contributed to our understanding of how neuropeptides regulate complex behavioral sequences (cf. Nässel, 2000; Predel and Eckert, 2000).

Insects have a rigid exoskeleton (cuticle). Continuous growth therefore requires the periodic replacement of the cuticle of one stage with a larger one for the next stage. Molting, the process of making a new cuticle, culminates with ecdysis, a stereotyped sequence of behaviors used to shed the old cuticle from the previous stage. Whereas molting is regulated by steroid hormones, ecdysis behavior is controlled by several interacting neuropeptides that ensure that the behavior is only turned on at the end of the molt. From work conducted primarily in the moth, Manduca sexta, we know that ecdysis behavior is controlled by at least three distinct neuropeptides: eclosion hormone (EH), ecdysis triggering hormone (ETH), and crustacean cardioactive peptide (CCAP). By the simplest hypothesis, EH and ETH release and interactions restrict the occurrence of ecdysis to the end of the molt. EH released within the CNS then acts on the CCAP neurons, and it is the ensuing release of CCAP that is believed to initiate ecdysis behavior (for review, see Ewer and Reynolds, 2002).

Although this model is consistent with most of the available data, a number of observations suggest that the endocrine control 
of ecdysis occurs via more complex mechanisms. For example, although the $\mathrm{EH}$ neuropeptide was believed to be the key inducer of ecdysis, Drosophila lacking EH neurons survive until adulthood and express only relatively subtle defects at adult eclosion (McNabb et al., 1997). These and other findings (Park et al., 2003) indicate that the precise contributions to ecdysis control by individual neuropeptide regulators are not easily defined. They also suggest that additional neuropeptides may participate to create more exact and complex regulation of this essential insect behavior.

Here we used wild-type Drosophila larvae, as well as larvae lacking EH neurons, CCAP neurons, or both EH and CCAP neurons, to investigate the endocrine bases of the behavioral, hormonal, and developmental events that are expressed at larval ecdysis. Our findings clarify some of the hierarchical relationships between the neuropeptides that control these events and provide evidence for direct versus indirect involvement of particular neuropeptides in the control of specific components of ecdysis. In particular, we present evidence indicating that factors other than $\mathrm{EH}$ are also involved in ETH release and that $\mathrm{EH}$, heretofore considered to act upstream CCAP, may also have functions that are partially redundant with it.

\section{Materials and Methods \\ Fly strains and genetics}

All flies were raised at $25^{\circ} \mathrm{C}$ on standard cornmeal-agar media under a 12 $\mathrm{hr}$ light/dark regimen. Wild-type flies were from a Canton-S strain maintained in our laboratory; the cytoplasmic UAS-LacZ line was obtained from the Bloomington Drosophila Stock Center (Indiana University, Bloomington, IN), and the UAS-reaper (UAS-rpr) strain was obtained from H. Steller, (Howard Hughes Medical Institute, The Rockefeller University, New York, NY). EH-GAL4 flies were kindly provided by S. $\mathrm{McNabb}$ and J. Truman (University of Washington, Seattle, WA) (cf. McNabb et al., 1997); the CCAP-GAL4 line has been described previously (Park et al., 2003). Flies bearing both EH-GAL4 and CCAP-GAL4 were generated by standard recombination and are referred to here as $\mathrm{EH}+\mathrm{CCAP}-\mathrm{GAL} 4$. Because driving LacZ expression in the $\mathrm{EH}$ and CCAP neurons produced $\beta$-galactosidase immunoreactivity (IR) that was stronger than the normal EH- and CCAP-IR, we used LacZ as an independent marker for the presence of these neurons. Thus, for experiments involving the targeted ablation of EH and/or CCAP neurons, we used flies bearing both UAS-rpr and UAS-LacZ inserts, which were generated by standard recombination, and are referred to here as UAS-rpr + LacZ. Targeted ablation of EH and/or CCAP neurons was produced by crossing flies bearing the appropriate GAL4 transgene to flies bearing UAS- $r p r+$ LacZ. In our hands, $100 \%$ of all EH neurons $(n=20)$ and $>99 \%$ of CCAP neurons and their processes $(n=20)$ were undetectable by midway through the second larval instar stage, as described previously using the CCAP-GAL4 line (Park et al., 2003). The absence of the relevant neurons was confirmed directly for all of the larvae used in this study. Controls were obtained by crossing flies bearing the same GAL4 drivers to UAS-LacZ flies.

\section{Behavioral analyses}

Larvae. Zero- to 12-hr-old first instar larvae were collected and reared at $25^{\circ} \mathrm{C}$ as described by Park et al. (2003). Larvae approaching the ecdysis from the second to the third instar were identified on the basis of the appearance of "double vertical plates" in the mouthparts (DVP stage; $\sim 30$ min away from ecdysis) (Park et al., 2002). Larval behavior was recorded at $25^{\circ} \mathrm{C}$ under a Leica (Nussloch, Germany) dissecting microscope using an Optronix 750DE camera attached to a Panasonic (Secaucus, NJ) AG-6040 time-lapse video recorder (used at normal speed). Scoring and quantitation of behavior was done by analyzing these recordings. Time of ecdysis was taken as the time of the third consecutive anteriorly directed ecdysis peristalsis wave.

Hormone injections. Drosophila melanogaster (Dm) ETH1 (ETH1) and DmETH2 (ETH2) were kindly provided by Y. Park (Kansas State Uni- versity, Manhattan, KS) and M. Adams (University of California, Riverside, CA) (cf. Park et al., 1999); synthetic Manduca sexta ETH (MasETH) was generously provided by A. Novicki (Johnson C. Smith University, Charlotte, NC); 8-Br-cGMP was obtained from Sigma (St. Louis, MO). Stock solutions of ETH hormones (1 mM) and of 8-Br-cGMP (30 mM) were prepared in distilled water, aliquoted, and stored at $-20^{\circ} \mathrm{C}$. Injection mixtures were prepared fresh on the day of the experiment by diluting the stock solutions using distilled water; $0.5 \%$ green food dye was included to aid in monitoring the success of the injection. For control (vehicle) injections, distilled water and dye was used. Injections were done into DVP stage larvae, using a pulled borosilicate glass microelectrode and a PV800 pneumatic picospritzer (World Precision Instruments, Sarasota, FL); the volume of the injection was adjusted for each needle to $\sim 10 \mathrm{nl}$ on the basis of the length traveled by the solution along the needle after 100-200 pulses. Larvae that were not successfully injected, that were damaged during the injection, or in which the injection was made into the gut, were discarded.

\section{Immunohistochemistry}

Tissue processing. Immunohistochemistry was used to determine the levels of EH and CCAP in the CNS and those of ETH in the seven pairs of peritracheal Inka cells and was performed essentially as described by Park et al. (2003) with a few modifications, as detailed below. Briefly, CNSs and the main tracheal trunks (which bear the ETH-containing Inka cells) were dissected under ice-cold PBS, fixed in $4 \%$ buffered paraformaldehyde fixative for $>12 \mathrm{hr}$ at $4^{\circ} \mathrm{C}$, and kept in cold fixative until processing. Care was taken to obtain intact pairs of tracheae, and, for each larva, $88-100 \%$ of the seven pairs of Inka cells present in each larva were recovered, all on a single piece of tissue. Tissues for a complete timeexperimental series $(8-10$ samples per time and dose, in addition to the relevant controls) were accumulated in cold fixative over the course of up to 1 week and then processed in parallel. For this, they were rinsed several times in PBS with $0.3 \%$ Triton X-100 (Sigma) (PBSTX) and incubated in primary antibodies for $>6 \mathrm{hr}$ at room temperature or overnight at $4^{\circ} \mathrm{C}$. The tissues were then rinsed several times in PBSTX and incubated in fluorescently tagged secondary antibodies (Jackson ImmunoResearch, West Grove, PA) for $>2 \mathrm{hr}$ at room temperature or overnight at $4^{\circ} \mathrm{C}$. Tissues were then rinsed several times in PBSTX, then PBS, and were mounted onto poly-L-lysine-coated coverslips, dehydrated, and mounted in DPX.

Primary antibodies were rabbit anti-CCAP (used at 1:5000; a kind gift from H.-J. Agricola, Jena University, Jena, Germany) (cf. Ewer and Truman, 1996), rabbit anti-ETH1 (used at 1:2000; a kind gift from M. Adams and Y. Park) (cf. Park et al., 2002), rabbit anti-EH (used at 1:150; kindly provided by J. Truman, University of Washington, Seattle, WA), sheep anti-cGMP (used at 1:500) (Baker et al., 1999), and mouse anti-LacZ (used at 1:2000; Promega, Madison, WI). In some early experiments, rabbit anti-myomodulin (kindly provided by K. Weiss, Mt. Sinai School of Medicine, New York, NY) (Miller et al., 1991), which cross-reacts with ETH (Zitnan et al., 2003), was used to monitor ETH release (cf. O'Brien and Taghert, 1998). All of the results reported here were obtained using the anti-ETH1 antibody. Preparations were viewed under a conventional fluorescent microscope as well as under a confocal microscope [Bio-Rad (Hercules, CA) MRC600 with a Nikon (Tokyo, Japan) inverted microscope or a Leica DMR system].

Quantitation of immunolabeling. CCAP- and ETH-IR were quantitated assigning a subjective score of 0 (no staining) to 3 (strongest staining). cGMP-IR showed less gradations in intensity and was scored qualitatively between 0 (no staining) and 2 (maximum staining). The person scoring the preparations did not know the time points at which the tissues had been fixed, with the exception of tissues dissected at DVP stage, which served as the reference for maximal CCAP and ETH staining for that experimental series. Immunoreactivity in each Inka cell was given a separate ETH and cGMP score; CCAP-IR was scored on the basis of the intensity of immunostaining of the lateral axons (see Fig. 1C).

EH-IR was scored as described by Husain and Ewer (2004). Briefly, Z-series of confocal sections were collected using the same nonsaturated settings for all preparations within an experimental series. They were then collapsed keeping the maximum intensity pixels, and the resulting 

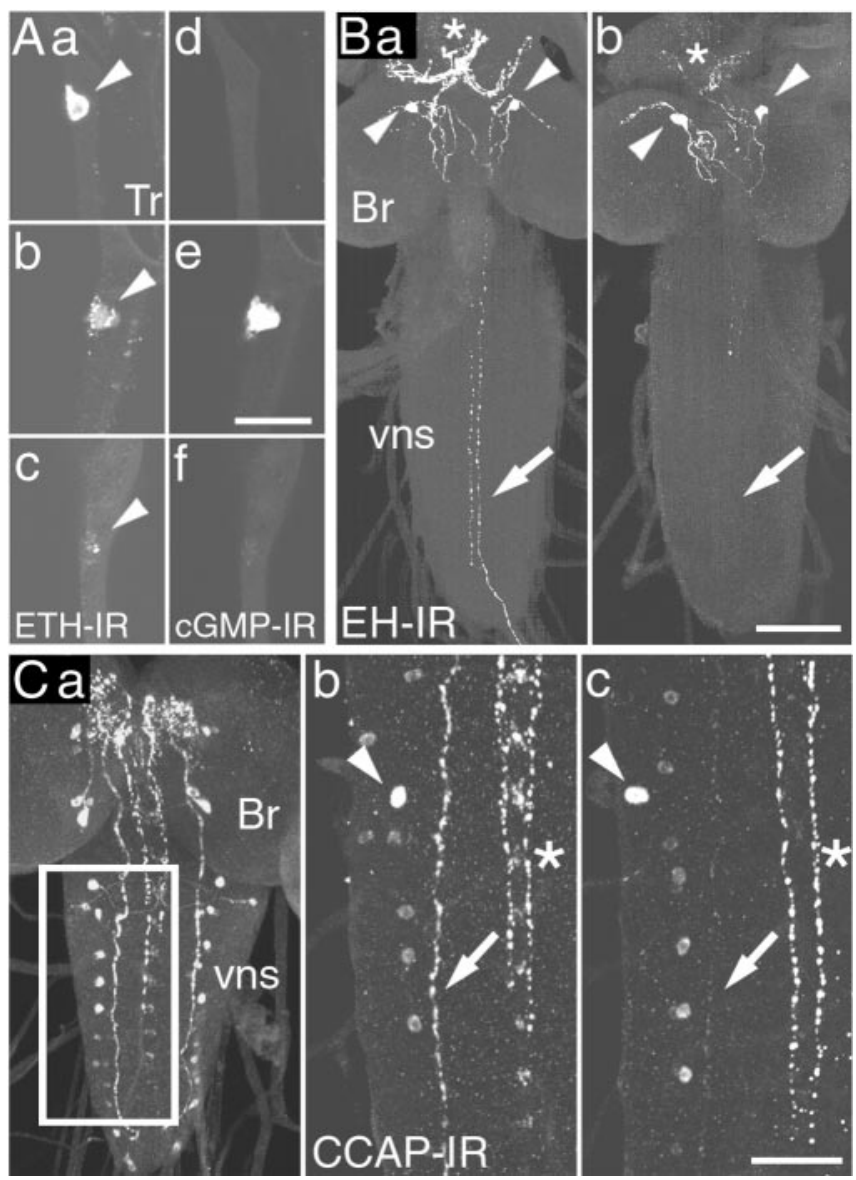

Figure 1. Neuropeptide release at larval ecdysis. A, ETH, Each pair of panels shows the pattern of ETH-IR ( $a-c$ ) and corresponding CGMP-IR $(d-f)$ in Inka cells (arrowhead) dissected at DVP stage $(a, d)$, shortly before the onset of ecdysis $(b, e)$, and at ecdysis $(c, f) . B$, EH, At DVP stage ( $a$ ), strong EH-IR occurred in the axons (arrow) of EH-producing neurons (arrowheads) and in their neurohemal release site in the ring gland (asterisks). After ecdysis (b), EH-IR was reduced from these locations. C, CCAP, $a$, Pattern of CCAP-IR (here shown in the CNS of a late third instar larva) (cf. Ewer and Truman, 1996; Park et al., 2003). CCAP-IR in the area of the CNS boxed in $a$ is shown for the CNS of larvae dissected before $(b)$ and after (c) ecdysis. Note the reduction in (CAP-IR in the descending axon (arrows) after ecdysis (c), indicating that this neuropeptide was released from these axons at this time; arrowheads indicate cell bodies in similar positions, and the asterisks indicates the medial CCAP-immunoreactive axons. Br, Brain; vns, ventral nervous system; $T r$, trachea. Scale bars: (in $A e$ ) $a-f, 20 \mu \mathrm{m}$; (in Bb) $a, b, 40 \mu \mathrm{m}$; (in (c) $b, c, 20 \mu \mathrm{m}$.

images were then analyzed using NIH Image (http://rsb.info.nih.gov/ nih-image). First, the background signal was subtracted, and the resulting image was smoothened. A threshold was then set such that only the intensely stained $\mathrm{EH}$-immunoreactive varicosities were visible, and the number of varicosities was counted using the same threshold for all preparations. A "varicosity index" was defined on the basis of the number of varicosities per unit of axon length.

\section{Results}

\section{Neuroendocrine events at ecdysis in wild-type larvae}

In Drosophila, ETH is produced by seven pairs of epitracheal Inka cells (Fig. 1A), which are located close to the branch point of most of the transverse connective tracheal branches off of the main tracheal trunk (O'Brien and Taghert, 1998; Park et al., 2002). EH is produced by a single pair of ventral medial $(\mathrm{Vm})$ neurons in the brain, with axonal projections in the ventral nervous system (VNS) and a neurohemal release site in the ring gland (Fig. $1 B$ ) (Horodyski et al., 1993). CCAP-IR in the CNS of Drosophila larvae occurs in two pairs of neurons in the brain and $\sim 15$ pairs in the subesophageal ganglion and the VNS. In addition, prominent CCAP-IR occurs in medial and lateral axonal bundles (Fig. 1C) (Ewer and Truman, 1996; Park et al., 2003). Larvae approaching ecdysis from the second to the third (last) larval instar were selected on the basis of the appearance of pigmentation in the mouthparts of the future third instar (DVP stage) (Park et al., 2002). At various times after DVP stage, the larvae were dissected, and the trachea was processed for ETH- and cGMP-IR and the CNS was processed for EH- or CCAP-IR. As shown in Figure 1, ecdysis was accompanied by the release of ETH peptides (ETH1 and ETH2) (Park et al., 1999) from the peripherally located epitracheal Inka cells into the circulation (cf. Park et al., 2002) and of central EH (cf. Horodyski et al., 1993) and CCAP into the circulation and the CNS. At the DVP stage, strong ETH-IR was distributed throughout the cytoplasm of the Inka cells (Fig. 1 $A a$ ). ETH-IR then became varicose before the onset of ecdysis behavior (Fig. $1 A b$ ), implying that ETH release had started. This change in ETH-IR was typically accompanied by increases in immunoreactivity to the second messenger cGMP (Fig. 1 $\mathrm{Ae}$ ). Because cGMP is believed to mediate the actions of EH (Truman et al., 1979; Ewer et al., 1997; Kingan et al., 1997, 2001) (for review, see Ewer and Reynolds, 2002), this increase in cGMP-IR suggests that $\mathrm{EH}$ was present in the hemolymph at this time. By the onset of ecdysis behavior, ETH-IR had reached its lowest level (Fig. $1 A c$ ), and the cGMP response had been extinguished (Fig. $1 A f)$. Within the CNS, ecdysis was accompanied by a decrease in EH-IR from the descending axons (Fig. $1 B$, compare $a, b$, arrows) and from the neurohemal release site of the EH-producing neurons (Fig. $1 B$, compare $a, b$, asterisks). The prominent lateral CCAP-immunoreactive axons lost much of their CCAP-IR at ecdysis (Fig. $1 C$, compare $b, c$, arrows), revealing that CCAP was also released during the expression of this behavior in the larva, as occurs at pupal ecdysis (Park et al., 2003).

\section{Timing of behavioral and developmental events at ecdysis in wild-type larvae}

The timing of ecdysis is tightly coordinated with the progression through the molt. Figure $2 \mathrm{~A}$ shows the sequence of morphological and behavioral events expressed by wild-type larvae as they approached ecdysis to the third larval instar (cf. Park et al., 2002, 2003). Approximately 15 min after DVP stage, the trachea started to buckle because of the collapse of the second instar tracheal lining (Fig. 2A, Collapse). Shortly after this, the trachea of the future third instar began to fill with air, typically starting at the posterior end of the larva, and was completed in one of the branches $\sim 1$ min later (Fig. $2 A$, Air). Both tracheae filled within $1 \mathrm{~min}$ of each other. Tracheal air filling was followed (but never preceded) by the onset of the preparatory behavior called preecdysis (Fig. $2 A$, gray section). This behavior lasted for $\sim 12 \mathrm{~min}$ and was terminated by the onset of "biting behavior," during which the larva appeared to be attempting to tear the anterior end of the old cuticle (Fig. 2A, Bite), which was followed shortly thereafter by the onset of ecdysis behavior (Fig. 2A, Ecdysis).

\section{Temporal relationship between behavioral and endocrine events at ecdysis in wild-type larvae}

Figure 2 shows the temporal relationship between the behavioral and physiological events that were expressed at larval ecdysis (Fig. $2 A$ ) and the underlying endocrine events (Fig. $2 B, C$ ). The earliest marker for the imminent onset of ecdysis behaviors was the collapse of the lining of the second instar trachea. Consistent with previous reports (Park et al., 2002), we found that ETH release was tightly correlated with this event and was essentially complete 
A

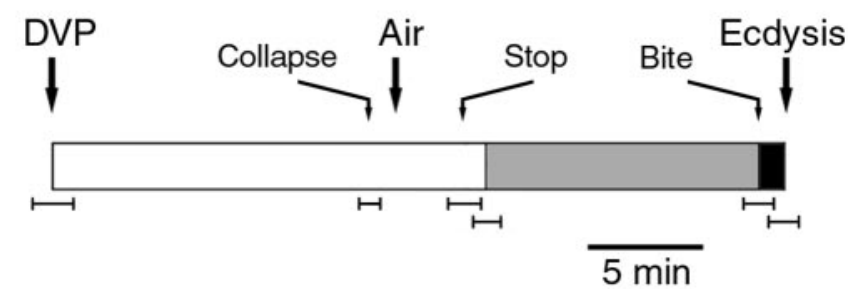

B

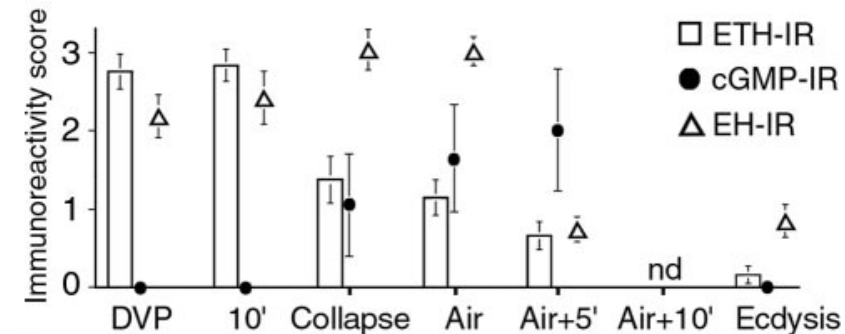

C

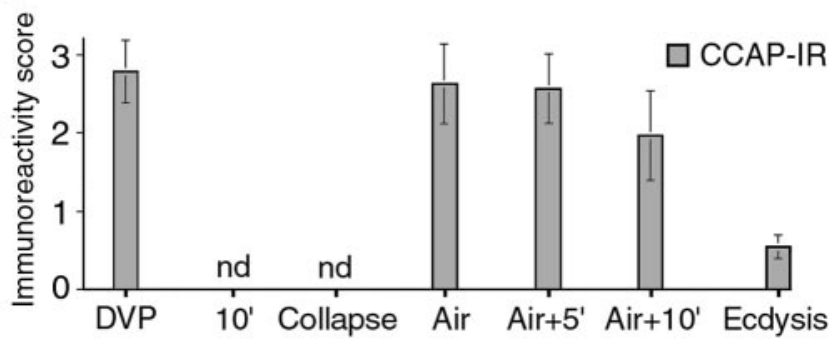

Figure 2. Time course of the physiological, behavioral, and endocrine events that occur at ecdysis from the second to the third larval instar in wild-type larvae. A, Open bars, Period between DVP stage and onset of pre-ecdysis (gray bar). Pre-ecdysis terminated with the onset of "biting" behavior (Bite), which was then followed by the initiation of ecdysis behavior (black bar). Additional events that occurred during the progression to ecdysis are also indicated. They were as follows: the collapse of the second instar trachea (Collapse); the entry of air into the third instar trachea (Air); and locomotion stopping (Stop). B, Immunoreactivity to ETH (open bars) and cGMP (filled circles) in Inka cells and to EH (open triangles) and CCAP-IR ( $C$ ) in the CNS, during the progression to ecdysis. The abscissa indicates the times at which larvae were examined relative to the events shown in $A$. In $A$, records from individual larvae were aligned relative to the time of air filling (Air) (average \pm SEM time for each event calculated). The SEM is indicated by error bars beneath the record; because Air corresponded to time 0 for all of the records, it had no associated error. Values in $B$ and C are average \pm SEM immunoreactivity scores ( $n>8$ for each data point). With the exception of $E H$, the quantitation of immunoreactivity was categorical; the SEMs associated with ETH- and CGMP-IR are only shown to illustrate the variance of the data and were not used to infer statistical significance. nd, Not determined.

5 min later. Although no significant changes in EH-IR were detectable at tracheal collapse, Inka cells showed increases in cGMP-IR at this time. Because EH is believed to act via cGMP (Truman et al., 1979; Ewer et al., 1997; Kingan et al., 1997, 2001) (for review, see Ewer and Reynolds, 2002) and increases in cGMP-IR in Inka cells did not occur in the absence of EH neurons (see below), the contemporaneous presence of cGMPimmunopositive Inka cells suggests that $\mathrm{EH}$ had also started to be released at this time. A fall in EH-IR was readily detectable 5 min after tracheal air filling (Air $+5^{\prime}$ ) and showed no additional change until the onset of ecdysis. Finally, the drop in CCAP-IR was not detected until shortly before the onset of ecdysis (Fig. 2C).

Role of EH in the control of ETH release: insights from intact wild-type larvae

The rise in CGMP-IR in Inka cells provided a sensitive monitor for the presence of EH in the hemolymph (Fig. $2 \mathrm{~B}$, filled circles).
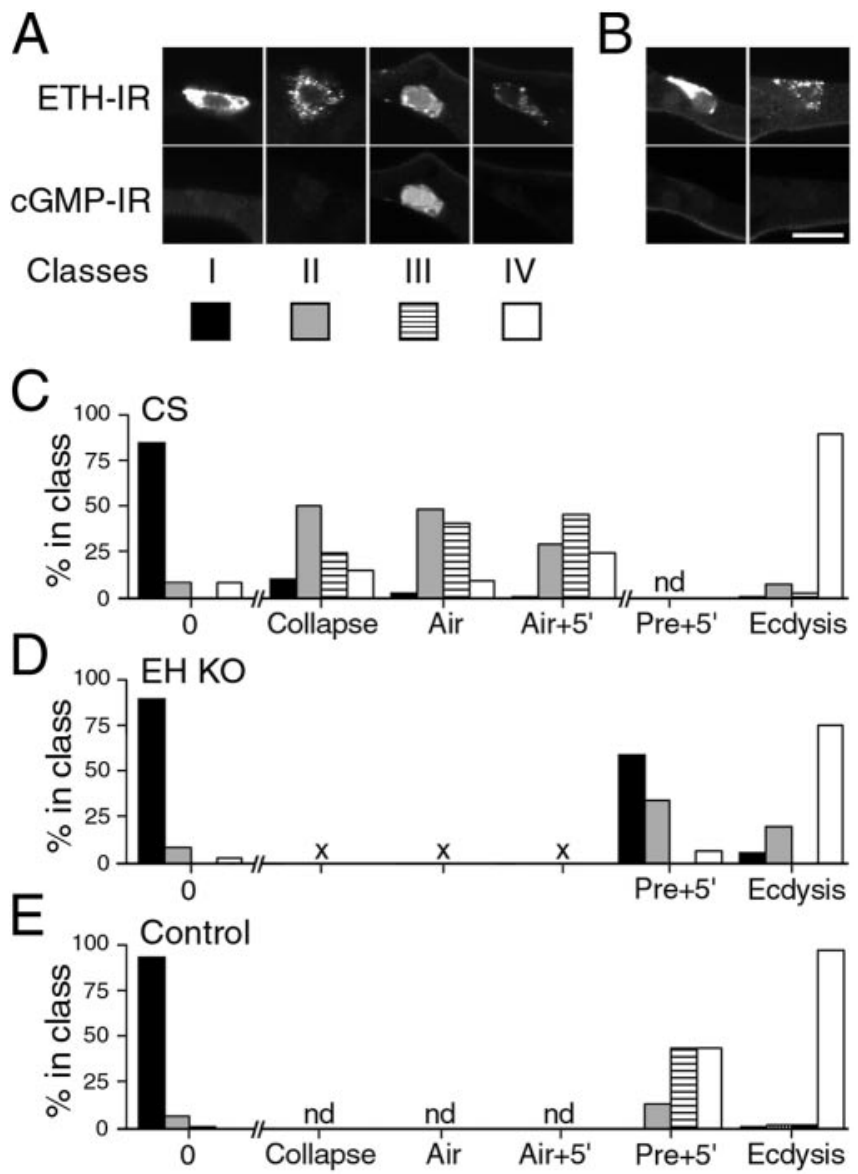

Figure 3. Relationship between ETH secretion and CGMP response in Inka cells at larval ecdysis. $A$, Examples from wild-type larvae of Inka cells in classes I-IV, showing ETH-IR (top) and corresponding CGMP-IR (bottom). $B$, Examples of two Inka cells from a single trachea of a EH-KO larva dissected at ecdysis (top row, ETH-IR; bottom row, corresponding CGMP-IR). (-E, Frequency of Inka cell classes I-IV at various times during progression to ecdysis in wild-type [C, CS (wild-type Canton-S strain)], EH KO (D, EH KO), and control ( $E$, Control) larvae. Each class is represented as indicated in $A$. The times examined correspond to times used in Figure 2 . nd, Not determined. $\times$ symbol in $D$ indicates that these time points could not be determined because of abnormal tracheal air filling in EH KO larvae (see Results). $n>9$ larvae for each time point. Scale $\operatorname{bar}$ (in $B$ ): $A, B, 10 \mu \mathrm{m}$.

To examine more closely the relationship between ETH and EH release, we classified Inka cells on the basis of their secretory state and cGMP response. Cells were scored blind and assigned to four classes, I-IV. Examples of these classes are shown in Figure $3 A$. Class I contained cells fixed before the start of ETH secretion and before the onset of a cGMP response. Cells in this class had ETH-IR and cGMP-IR scores of 3 and 0, respectively, and ETH-IR was distributed uniformly throughout the cytoplasm of the cell (Fig. 1 $\mathrm{Aa}$ ). Class II contained Inka cells that displayed varicose ETH staining (score of 2 or 1 ) but lacked a cGMP response (score of 0 ). Inka cells in class III had the same range of ETH-IR scores as those in class II but expressed a cGMP response (Fig. $1 A b, A e$ ). Finally, Inka cells that were essentially devoid of ETH-IR (score of 0 ) and showed no cGMP-IR (score of 0 ) were assigned to class IV (Fig. $1 A c, A f$ ).

Figure $3 C$ shows the relative distribution of each class of Inka cell in wild-type larvae fixed between the DVP stage and ecdysis. As expected on the basis of Figure $2 B$, most Inka cells from larvae fixed at the time of tracheal collapse were secreting ETH, because only $10.6 \%$ were in class I at this time $(n=123$ cells; representing $97 \%$ of all cells present in the nine larvae examined for this time 
point). Surprisingly, half (50.4\%) of the cells from larvae fixed at this time point belonged to class II, indicating secretory activity without a detectable cGMP response. The proportion of cells in this class then decreased over time, as the fraction of classes III and IV increased. By the time ecdysis behavior began, the majority of the Inka cells (89\%) became devoid of ETH staining and showed no cGMP staining (class IV) $(n=111$; representing $99 \%$ of all cells in the eight larvae examined at this time point).

Finding a large fraction of Inka cells in class II during the initial period of ETH secretion suggested that ETH release could occur in the absence of EH action. Alternatively, it could be that the EH-induced cGMP increase was short-lived and had already been effected by this time. Although this possibility cannot be completely ruled out, the time course of changes in classes II and III is not consistent with this interpretation. Indeed, if the cGMP response were very short-lived, the percentage of cells in class III would always be a fraction of the percentage of cells in class II, because it would represent the subset of cells that had been fixed just at the moment of the short-lived cGMP response. Instead, Figure $3 C$ shows that the fraction of class III Inka cells increased from 24.4 to $45.9 \%$ between tracheal collapse and Air +5 min, whereas that in class II fell from 50.4 to $28.9 \%$ during this same interval.

\section{Role of EH in the control of ETH release: insights from intact EH knock-out larvae}

To further investigate the role of EH in ETH secretion, we examined the time course of ETH release in larvae lacking EH neurons [EH knock-out (KO) larvae]. In these larvae, targeted ablation of $\mathrm{EH}$ neurons has been accomplished (and confirmed; see Materials and Methods) by expressing the Drosophila cell death gene reaper specifically in these neurons (McNabb et al., 1997). Figure $3, D$ and $E$, shows the distribution of Inka cells belonging to classes I-IV at DVP stage, 5 min after the onset of pre-ecdysis, and at ecdysis in $\mathrm{EH} \mathrm{KO}$ (Fig. 3D) and in the appropriate control larvae (Fig. 3E). [EH KO larvae did not show normal tracheal air filling (see below), so the earlier time points used in Figures 2 and $3 C$ could not be identified in EH KO larvae.] There were several notable features to the temporal pattern of ETH release seen in EH KO larvae (Fig. $3 B, D$ ). First, a significant fraction of Inka cells were clearly releasing (or had released) ETH during the expression of pre-ecdysis ( $40.9 \%$ of cells examined were in classes II-IV; $n=369$, corresponding to $85 \%$ of all cells from 31 larvae fixed at this time point). This was expected given that the ETH peptides are believed to play a key role in turning on pre-ecdysis behavior (Zitnan et al., 1996; Park et al., 2002) (for review, see Ewer and Reynolds, 2002). However, detecting ETH release at this time indicates that ETH can be released in the absence of EH, consistent with our findings in wild-type larvae (Fig. $3 C$, class II). Second, the distribution of Inka cells in the different classes during pre-ecdysis was strikingly different from that seen in controls (Fig. $3 E$ ), with a significant fraction of Inka cells showing no ETH release at this time (class I, 59.1\%). Even at ecdysis, some Inka cells either showed no sign of changes in ETH staining or were in the initial phase of change (5.9 and $19.5 \%$ of cells were in classes I and II, respectively; $n=256$, representing $91.4 \%$ of cells from 20 larvae dissected at ecdysis) (Fig. 3B). Finally, no cGMP response was ever detected in EH KO larvae (Fig. 3D, class III), supporting the hypothesis that this response is caused by the action of circulating EH on the Inka cells. In control larvae (Fig. $3 E$ ), the relative proportion of Inka cells seen in each class at the three times examined was consistent with the time course shown for wildtype larvae (Fig. 3C).

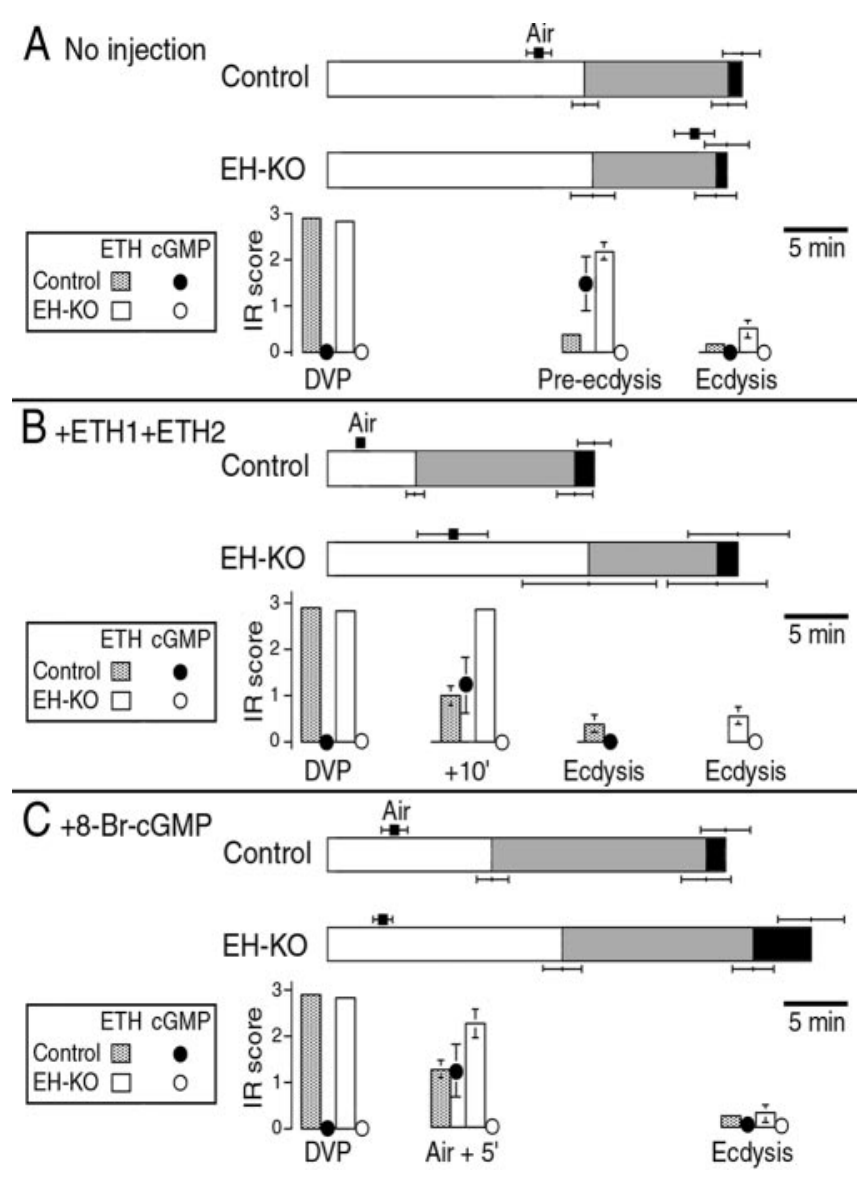

Figure 4. Time course of the behavioral and endocrine events that occur at larval ecdysis in intact and hormone-injected control (Control) and EH KO (EH-KO) larvae. A, Behaviors expressed at ecdysis by intact larvae (horizontal bars) and levels of ETH-IR and corresponding CGMP-IR in Inka cells from larvae fixed at the times indicated (histograms). Coding of the behavior is that used in Figure 2, except that the average time to tracheal air filling is indicated by a black square above the record of the behavior. B, C, Behavior (horizontal bars) and ETH-IR in Inka cells and corresponding CGMP-IR (histograms), summarized as in $A$, in larvae injected with ETH1 + ETH2 $(B, \mathrm{ETH} 1+\mathrm{ETH} 2 ; 1000 \mathrm{fmol}$ each) or 8-Br-CGMP (C, $+8 \mathrm{Br}-\mathrm{CGMP} ; 3 \mathrm{pmol})$. Times indicated are averages $\pm \mathrm{SEM} ; n>8$ larvae per data point.

\section{Timing of behavioral and developmental events in larvae lacking EH neurons}

To investigate the role of $\mathrm{EH}$ at ecdysis, we next examined the timing of the events shown in Figure $2 \mathrm{~A}$ in $\mathrm{EH} \mathrm{KO}$ larvae versus that in the appropriate control larvae. Because the $\mathrm{EH} \mathrm{KO}$ larvae did not show normal tracheal air filling (see below), the two records were aligned relative to the DVP stage. As illustrated in Figure $4 A$, and detailed in Figure 5 (Vehicle), we found no statistically significant difference between EH KO (label: KO) and control larvae (label: $C$ ) in the length of the interval between DVP stage and the start of pre-ecdysis (Fig. $5 B$ ), in the duration of pre-ecdysis (Fig. 5C, Duration), or in the time from DVP stage to ecdysis (Fig. $5 D)$ ( $p>0.1$ for each comparison; Student's $t$ test). Thus, the lack of EH neurons caused no significant differences in the time of onset and duration of pre-ecdysis and ecdysis. These results imply that the threshold level of ETH needed to cause pre-ecdysis was reached at the same time regardless of the presence of $\mathrm{EH}$. Nevertheless, these larvae were severely defective in tracheal air filling (Figs. 4A, 5A). All EH KO larvae included in these summaries were able to fill at least one trachea by the time of ecdysis. However, while selecting these larvae for testing, we noticed that some had died shortly after ecdysis, and all showed 
completely uninflated tracheae. As noted previously (McNabb et al., 1997), this suggests that the lethality associated with the lack of EH neurons may be caused primarily by respiratory failures rather than by an inability to shed the old cuticle. In addition to defects in tracheal inflation, the strength of pre-ecdysis behavior of EH KO larvae, as measured by the number of pre-ecdysis contractions expressed (Fig. 5C, Frequency), was significantly lower in $\mathrm{EH} \mathrm{KO}$ compared with that seen in control larvae $(p<0.05$; Student's $t$ test) (Fig. 5C).

The defects in tracheal air filling and pre-ecdysis strength in $\mathrm{EH} \mathrm{KO}$ larvae could implicate EH directly in the control of these processes. Alternatively, it could be that these defects are an indirect consequence of the abnormal time course of ETH release that occurs in these larvae (Figs. 3D, 4A). To distinguish between these possibilities, we injected $\mathrm{EH} \mathrm{KO}$ larvae with suprathreshold amounts of ETH1 and ETH2 or with a membrane-permeable analog of cGMP (8-Br-cGMP), which mimics the action of $\mathrm{EH}$ (Kingan et al., 1997, 2001; Husain and Ewer, 2004) (for review, see Ewer and Reynolds, 2002). We then examined the timing of air filling, pre-ecdysis, and ecdysis, as well as that of ETH release and (in control larvae) that of EH release monitored via the presence of cGMP-IR in the Inka cells. These results are summarized in Figure 4, $B$ and $C$, for ETH1+ETH2 and 8-BrcGMP injections, respectively. The duration, latency, and frequency of the events associated with individual segments of ecdysis behaviors are summarized in Figure 5.

Preliminary experiments using wild-type larvae established that the doses of ETH1 and ETH2 needed to elicit robust responses were $\sim 0.1$ and $10 \mathrm{fmol}$, respectively, consistent with previous reports (Park et al., 2002). Tests using different concentrations of these hormones (spanning 0.1-1000 fmol/injection) failed to reveal any qualitative differences in the relative ability of these peptides, either alone or in combination, to cause premature tracheal air filling, onset of pre-ecdysis and ecdysis, or release of the endogenous stores of ETH and EH (A. C. Clark and J. Ewer, unpublished observation). It therefore appears that the Drosophila ETH peptides both cause the same effects but do so with significantly different potencies (cf. Park et al., 1999). This situation is similar to that in the moth, Bombyx mori (Zitnan et al., 2002), but differs from what is found in the hornworm, Manduca sexta, in which pre-ecdysis triggering hormone, one of the two ETH peptides, can only elicit pre-ecdysis behavior regardless of the dose of hormone injected (Zitnan et al., 1999).

Injection of ETH1+ETH2 (mixtures of either 5 fmol each [Fig. 5, ETH1/2 (low)] or $1000 \mathrm{fmol}$ each [Figs. 4B, 5, ETH1/2 (high)] of each peptide were tested) caused a significant shortening in the latency to ecdysis in control larvae because of the premature onset of all of the behavioral and developmental events expressed at this time. Interestingly, the duration of pre-ecdysis was not significantly different between vehicle-injected and $\mathrm{ETH} 1+\mathrm{ETH} 2$-injected control larvae $(p>0.05$ for 5 and 1000 fmol mixtures; ANOVA) (Fig. $5 C$ ), indicating that the shorter time to ecdysis of ETH-injected control larvae was attributable to

\section{B Pre-ecdysis start}

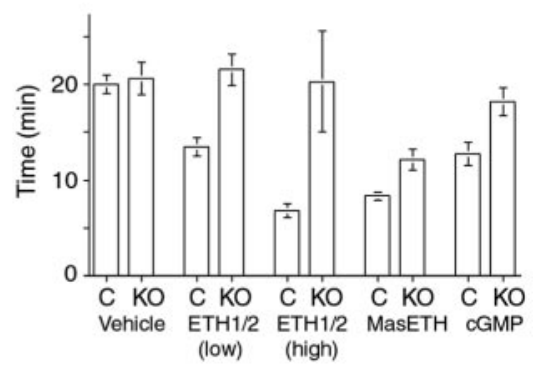

D Ecdysis start

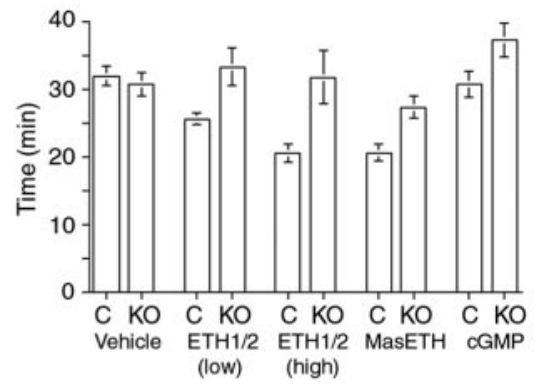

First trachea Second trachea

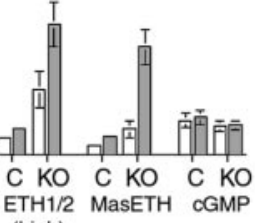
igh)
Figure 5. Behavioral responses to hormone injection in control larvae (label: C) and in EH KO larvae (label: K0). Each histogram
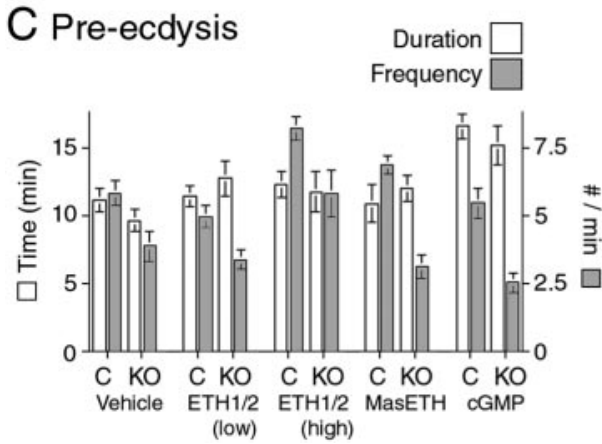

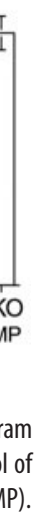
P
of
P). MP). $\mathrm{ETH} 1+\mathrm{ETH} 2$ [ETH1/2 (low)], $1000 \mathrm{fmol}$ of ETH1+ETH2 [ETH1/2 (high)], $1000 \mathrm{fmol}$ of MasETH, or 3 pmol of 8-Br-cGMP (cGMP). Values are averages \pm SEM ( $>8$ larvae per data point).

a reduction in the latency to start the pre-ecdysis behavioral sequence. Injection of these peptides also caused a premature release of the endogenous stores of ETH and onset of the cGMP response (compare ETH- and cGMP-IR at $10^{\prime}$ in Figs. $2 \mathrm{~B}, 4 \mathrm{~B}$ ). In contrast, injection of ETH1+ETH2 (5 or $1000 \mathrm{fmol}$ of each peptide) had only slight effects on EH KO larvae (Figs. $4 B$, 5). Even at the highest doses tested (1000 fmol of each peptide), there was no consistent premature onset of pre-ecdysis and ecdysis behaviors and no premature release of ETH (Figs. $4 B, 5$ ). Although the behavioral responses of EH KO larvae to ETH injections were quite variable, statistically they were not significantly different from those expressed by control EH KO larvae $(p>0.05$; ANOVA). The inability of ETH peptides to consistently cause the premature ecdysis of $\mathrm{EH} \mathrm{KO}$ animals has been noted previously for adult eclosion (McNabb et al., 1997; Baker et al., 1999) using either Manduca Inka cell extract or MasETH. Here we also tested synthetic MasETH (1000 fmol dose) and found that it was able to accelerate the onset of pre-ecdysis in EH KO larvae $(p<0.01$; Student's $t$ test) but not to the extent seen in control larvae $(p<$ 0.05 ; Student's $t$ test) (Fig. $5 B$, MasETH). Despite the premature onset of pre-ecdysis, MasETH-injected EH KO larvae expressed a longer pre-ecdysis than did vehicle-injected EH KO larvae, resulting in no net shortening in the time between DVP stage and ecdysis ( $p>0.05$; Student's $t$ test) (Fig. $5 D$ ). Given the inability of Drosophila ETH peptides to cause the premature ecdysis of EH $\mathrm{KO}$ larvae, the responsiveness of these larvae to the heterologous MasETH peptide is difficult to explain and will have to await structure-function analyses of these hormones.

In $\mathrm{EH} \mathrm{KO}$ larvae, the strength of pre-ecdysis (on the basis of the number of pre-ecdysis contractions per minute) was not increased by injections of ETH1+ETH2 [5 fmol each; Fig. 5C, ETH1/2 (low)] or MasETH (1000 fmol; Fig. 5C, MasETH). Although the higher dose of ETH1+ETH2 [1000 fmol each; Fig. 
5C, ETH1/2 (high)] did increase the strength of pre-ecdysis behavior to normal levels (those expressed by intact or vehicleinjected control larvae), it was significantly weaker than that of control larvae injected with this high dose of hormones $(p<$ 0.05; Student's $t$ test) [Fig. 5C, ETH1/2 (high)]. Overall, these results reveal that the defects expressed by EH KO larvae were not attributable simply to the abnormal timing of ETH release, because they were not rescued by the injection of suprathreshold amounts of ETH peptides.

Injection of ETH1+ETH2 was able to induce premature air filling in $\mathrm{EH} \mathrm{KO}$ larvae but never as effectively as occurred in control larvae. As shown in Figure $4 B$, and as detailed in Figure $5 \mathrm{~A}$, the latency to fill the first trachea was greater in $\mathrm{ETH} 1+\mathrm{ETH} 2$-injected EH KO animals than it was in hormoneinjected control larvae, and the lag time between the filling of the first and second trachea was always greater in EH KO larvae than in control larvae, even at the highest dose tested. Interestingly, MasETH $(1000 \mathrm{fmol})$ was more effective than the fly peptides when tested at the same high dose (Fig. 5A, MasETH). Nevertheless, this peptide was also unable to effect the rapid and complete air filling obtained in the control larvae.

Injections of 8-Br-cGMP ( $3 \mathrm{pmol})$, in contrast, caused a rapid and complete tracheal air filling in EH KO larvae that was indistinguishable from the response seen in control larvae (Figs. 4C, $5 A, \mathrm{cGMP})$. Not only did the tracheae fill within the same time as occurred in control larvae, but there was also no significant lag time between the filling of both tracheae. Thus, 8-Br-cGMP, which is believed to act as an EH analog (Kingan et al., 1997, 2001; Husain and Ewer, 2004) (for review, see Ewer and Reynolds, 2002), was able to completely rescue the tracheal defects seen in EH KO larvae. This membrane-permeable cGMP analog also caused some release of ETH in EH KO animals (as indicated by reduced ETH-IR) (Fig. 4C) although not to the extent seen in 8-Br-cGMP-injected control larvae. This difference may have been observed because, in the latter case, ETH release caused EH release (Fig. 4C, cGMP), and EH induces the secretion of ETH via two second-messenger systems, cGMP and $\mathrm{Ca}^{2+}$ (Kingan et al., 2001). Thus, EH may be more effective in causing ETH release than 8-Br-cGMP is on its own. Drosophila Inka cells challenged in vitro with 8-Br-cGMP also show only partial ETH release (Husain and Ewer, 2004), consistent with this interpretation.

Injections of 8-Br-cGMP caused a more complex behavioral response than did the ETH peptides. Although 8-Br-cGMP caused a premature onset of pre-ecdysis in control larvae $(p<$ 0.01; Student's $t$ test) (Figs. $4 C, 5 B$, cGMP), the behavior lasted longer than normal (Fig. 5C, cGMP), resulting in no net shortening in the time from DVP stage to ecdysis ( $p>0.05$; Student's $t$ test) (Fig. $5 D$, cGMP). In contrast to the response seen in control larvae, cGMP injections did not cause a premature onset of preecdysis in EH KO larvae ( $p>0.05$; Student's $t$ test) (Fig. $5 B$, cGMP). Nevertheless, these cGMP injections did cause a lengthening of pre-ecdysis (Fig. $5 C$, cGMP), thereby causing a significant increase in the time between DVP stage and ecdysis $(p<$ 0.05 ; Student's $t$ test) (Fig. 5D, cGMP).

\section{Evidence for degeneracy in the control of ecdysis by EH and CCAP}

Work in Manduca has suggested that CCAP is the key neuropeptide that turns on the ecdysis motor program (Gammie and Truman, 1997). Although CCAP was released shortly before the onset of larval ecdysis (Fig. 2C), we showed that the genetic ablation of CCAP neurons in Drosophila larvae causes only relatively minor, albeit statistically significant, behavioral defects in the ec-
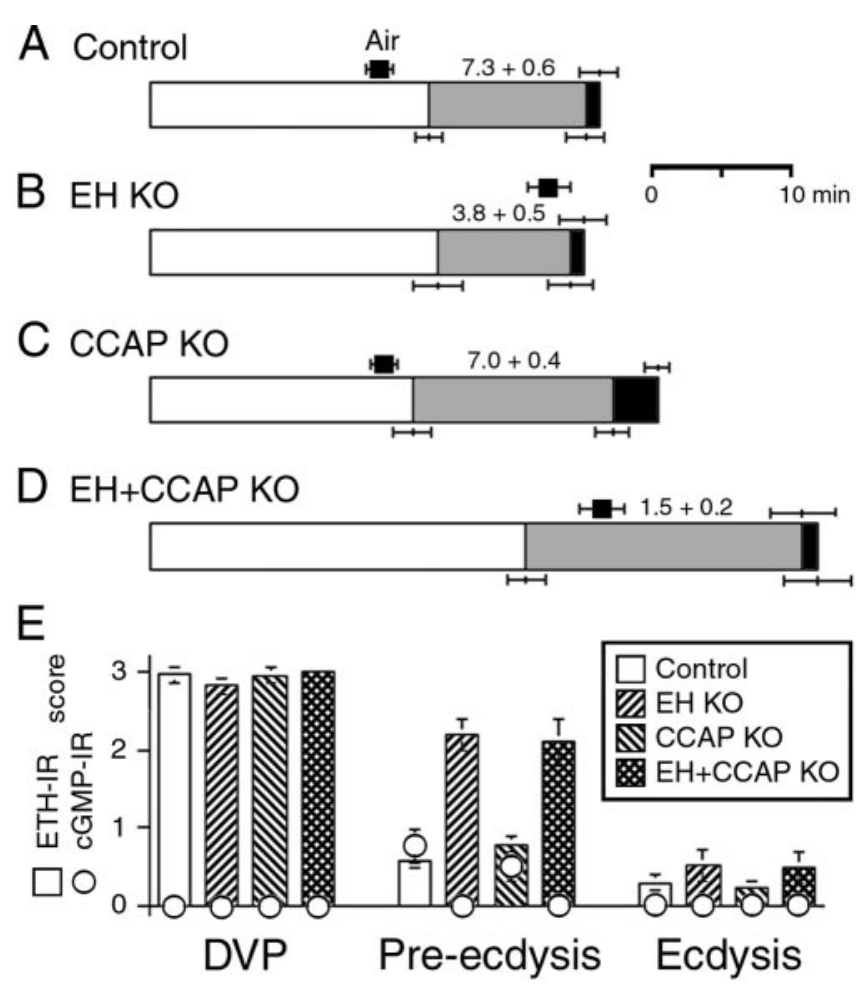

Figure 6. Time course of the behavioral $(A-D)$ and endocrine events $(E)$ that occur at larval ecdysis in intact control $(A)$, EH KO $(B)$, CCAP KO $(C)$, and EH + CCAP KO $(D)$ larvae. Control larvae were the progeny of EH + CCAP-GAL4 $\times$ UAS-LacZ. Behavior has been coded as explained in Figure 2, except that the average time to tracheal air filling is indicated by a black square above the record of the behavior. Numbers above the pre-ecdysis segment of the behavior represents the frequency of pre-ecdysis (contractions per minute). Values are averages \pm SEM ( $n>8$ larvae per data point).

dysis behaviors expressed at this stage (Park et al., 2003). Thus, pre-ecdysis is slightly longer in larvae lacking CCAP neurons (CCAP KO larvae), although the behavior is expressed with normal vigor (on the basis of the frequency of pre-ecdysis contractions). In addition, ecdysis behavior itself lasts approximately three times longer than in control larvae. Despite these abnormalities, $\sim 100 \%$ of CCAP KO larvae are able to shed their old cuticle at larval ecdysis, and the time between DVP stage and the shedding of the cuticle is only lengthened by $\sim 14 \%$ compared with that of the appropriate control larvae.

It could be that CCAP is released at larval ecdysis but plays no role in controlling ecdysis at that stage. Alternatively, there could be degeneracy in this system (cf. Edelman and Gally, 2001; Greenspan, 2001), with multiple peptides controlling this behavior. We wondered whether $\mathrm{EH}$ neurons could be involved in turning on ecdysis independent of CCAP. To test this hypothesis, we examined the events shown in Figure 2 in larvae lacking both $\mathrm{EH}$ and CCAP neurons (EH+CCAP KO larvae) and compared them with those expressed by EH KO and CCAP KO larvae and by the relevant control larvae. The results obtained are summarized in Figure 6 and reveal that EH +CCAP KO larvae expressed significantly more severe abnormalities at ecdysis than did $\mathrm{EH}$ $\mathrm{KO}$ and CCAP KO larvae. Furthermore, these abnormalities were typically more severe than the combination of the defects seen in larvae lacking either of these two neuronal populations. Thus, although EH KO and CCAP KO animals did not differ from control larvae in the time of onset of pre-ecdysis, the onset of this behavior was significantly delayed in EH + CCAP KO larvae compared with the times for these three genotypes $(p<0.05$; 
ANOVA). Likewise, the time from DVP stage to ecdysis was significantly longer in $\mathrm{EH}+\mathrm{CCAP} \mathrm{KO}$ compared with that of control, EH KO, and CCAP KO larvae $[p<0.05$ for each comparison with $\mathrm{EH}+\mathrm{CCAP} \mathrm{KO}$; ANOVA, followed by Tukey's honestly significant difference (HSD) post hoc test]. Finally, the strength of pre-ecdysis, measured as the rate of preecdysis contractions, was significantly lower in $\mathrm{EH}+\mathrm{CCAP} \mathrm{KO}$ larvae than it was in $\mathrm{EH} \mathrm{KO}$ animals [this parameter was normal in CCAP KO larvae (Fig. 6C)] (Park et al., 2003) ( $p<0.05$ for each comparison with $\mathrm{EH}+\mathrm{CCAP} \mathrm{KO}$; ANOVA, followed by Tukey's HSD post hoc test). In fact, the whole ecdysis sequence expressed by $\mathrm{EH}+\mathrm{CCAP} \mathrm{KO}$ larvae was weak enough to cause additional lethality at ecdysis over that seen in $\mathrm{EH} \mathrm{KO}$ animals. Survival from first to third instar was $98 \%$ in controls $(n=511), 31 \%$ in EH KO larvae $(n=260)$, and only $22 \%$ in $\mathrm{EH}+\mathrm{CCAP} \mathrm{KO}(n=740)$. As noted above, the vast majority of dead EH KO larvae had fluid-filled trachea, suggesting that the main cause of lethality in this genotype was attributable to the failure to correctly fill the new trachea with air. In addition to defects in tracheal inflation, many dead EH+CCAP KO larvae showed a "buttoned-up" phenotype (Park et al., 2002), indicating that they were unable to shed their old mouthparts (Fig. 7E) because of a failure to ecdyse normally.

The absence of CCAP neurons in addition to $\mathrm{EH}$ neurons did not appear to cause additional defects in the timing of ETH release than did the lack of only the $\mathrm{EH}$ neurons (Fig. 6E): $\mathrm{EH}+\mathrm{CCAP} \mathrm{KO}$ larvae dissected during pre-ecdysis showed a similar partial level of ETH release as that seen in EH KO animals, and larvae from both of these genotypes had released most of their stores of ETH at the time of ecdysis. A more detailed time course of ETH release may, however, reveal some differences in the exact timing of ETH release between these two genotypes.

\section{Molting defects in EH KO larvae}

In normal larvae, ecdysis is tightly coordinated with the molt cycle (Fig. 2). The most useful marker for the impending onset of the physiological and behavioral events expressed at ecdysis is the appearance of pigmentation in the mouth plates of the third instar (DVP stage). The strict timing of the ecdysis behaviors relative to this marker (Fig. $2 \mathrm{~A}$ ) results in third instars expressing similar levels of mouthpart pigmentation at the end of ecdysis (exemplified in Fig. 7A). The EH KO larvae used for the data reported here were selected at the DVP stage and showed levels of pigmentation comparable with those seen in control larvae. However, we noted that the population from which these larvae were selected could occasionally include recently ecdysed third instar larvae that had much lighter mouthpart pigmentation than normal (Fig. $7 B, C$ ). In addition to defects in overall pigmentation, differences in the amount of pigmentation between the two sides of the mouth plates were sometimes observed (Fig. 7D); such differences were never observed in control or wild-type larvae. Whereas the lighter pigmentation expressed by some $\mathrm{EH} \mathrm{KO}$ larvae after ecdysis (Fig. 7 B, C) suggests that ecdysis may have been turned on earlier than normal in $\mathrm{EH} \mathrm{KO}$ larvae, the differential tanning of the two sides of the mouthparts (Fig. 7D) suggests additional defects in the progression through the molt. The nature of these defects is currently unknown. It is noteworthy that larvae genetically lacking ETH are also reported to initiate ecdysis-like behaviors earlier than normal (Park et al., 2002).

\section{Discussion}

Ecdysis involves the expression of a series of behavioral and physiological events whose precise timing and concatenation is essential for insect survival. The stereotyped deployment of these events is controlled by several neuropeptides, whose exact time of release at the end of the molt is precisely regulated by their complex endocrine relationships (for review, see Ewer and Reynolds, 2002). Here, we examined the timing of the behaviors and physiological events expressed during larval ecdysis in Drosophila and correlated it with the timing of the underlying endocrine events to disentangle direct versus indirect neuropeptide actions. Our findings using wild-type larvae, as well as $\mathrm{EH} \mathrm{KO}, \mathrm{CCAP} \mathrm{KO}$, and $\mathrm{EH}+\mathrm{CCAP} \mathrm{KO}$ neurons, reveal a number of previously unsuspected features and relationships involved in the neuroendocrine control of ecdysis. These findings have been incorporated into a model for the neuropeptide control of ecdysis shown in Figure 8.

\section{Role of EH in the release of ETH}

Previous work using Manduca has shown that EH acts directly on Inka cells to induce ETH release (Ewer et al., 1997; Kingan et al., 1997, 2001) and that this action is mediated at least in part by cGMP (Kingan et al., 1997, 2001). Similarly, we showed here that ETH secretion in vivo is accompanied by increases in cGMP-IR and that cGMP increases are absent in EH KO larvae. These findings implicate EH in the process of ETH release in Drosophila and, furthermore, provide the first demonstration that the cGMP response seen in Inka cells in vivo is entirely mediated by the $\mathrm{EH}$ neurons. In a previous study, we showed that Drosophila Inka cells release ETH when challenged with 8-Br-cGMP in vitro, implicating cGMP in ETH secretion in the fly (Husain and Ewer, 2004).

Although these results suggest that EH can cause ETH release, we also found that EH was not necessary for ETH secretion. Instead, the role of EH appears primarily to be in causing the phasic and coordinated release of ETH that is seen in vivo. Our results suggest the following scenario: shortly before tracheal collapse, ETH starts to be secreted, much of it via an EH-independent mechanism (Fig. 8, [1]). Shortly after this, EH, likely released in 


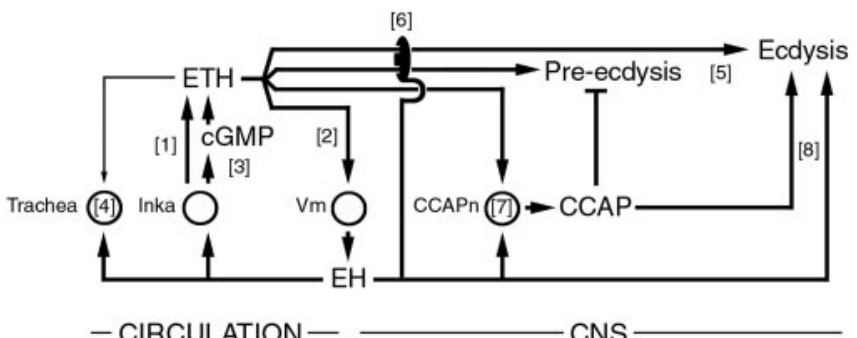

Figure 8. Model for actions and relationships among ecdysis-controlling neuropeptides. The endocrine cascade that turns on the ecdysis behaviors is likely initiated by an EH-independent ETH secretion from Inka cells ([1]). This release then causes secretion of EH from central Vm neurons ([2]), which stimulates additional ETH release via increases in CGMP ([3]). Circulating EH and ETH cause air filling of the trachea ([4]). ETH peptides also act on the CNS to turn on pre-ecdysis and ecdysis ([5]) (Park et al., 2002). However, these actions require EH neurons, because the ecdysis of EH KO larvae is not accelerated nor is the strength of pre-ecdysis restored by exogenous ETH ([6]). ETH and EH are involved in CCAP release from central CCAP neurons (CCAPn; [7]). CCAP inhibits pre-ecdysis (Park et al., 2003). ETH mutants show some ecdysis-like behaviors (Park et al., 2002) that could be controlled independently by EH and CCAP. Circles indicate known target cells. With the exception of [3], most actions are likely indirect. This model is primarily based on data from Drosophila and is consistent with information available from Manduca.

response to ETH (Fig. 8, [2]), causes further secretion of ETH (Fig. 8, [3]). The stimulation of ETH secretion caused by ETHinduced EH release would account for the rapid and almost complete depletion of ETH that occurs in wild-type larvae and explain the slower ETH release seen in EH KO animals.

\section{Precise timing of the initial ETH secretion}

A notable feature of ETH release is that the initial EHindependent secretion (Fig. 8, [1]) started at exactly the appropriate developmental time. How can such precise timing relative to the molt cycle be effected? Interestingly, not all Inka cells participated in this initial EH-independent ETH secretion. This was especially evident in EH KO larvae (Fig. 3D) in which, 5 min after the start of pre-ecdysis, only $\sim 40 \%$ of all Inka cells were releasing or had released ETH. This lack of synchrony among Inka cells suggests that the initial secretory phase is probably not caused by a sudden surge in a circulating factor. In addition, the Inka cells are not innervated (O'Brien and Taghert, 1998; Park et al., 2002), so this initial secretion would not be caused by neural stimulation. In vitro studies show that Manduca Inka cells can release some ETH in the absence of EH and that the rate of this release increases as the cells are taken from animals closer to the end of the molt (Kingan et al., 1997). The proximate cause of this secretory process is likely the fall in the titers in 20-hydroxy-ecdysone that occurs at the end of the molt, which also regulates the competence of Inka cells to respond to EH (Kingan and Adams, 2000). Whether the same process accounts for the initial, EHindependent, ETH secretion seen here is unknown. If it were, it would imply that these titers fall with an extremely precise time course. Alternatively, it could be that ETH release is initiated in some cells as a result of chemical or mechanical changes in their local environment that are correlated with the final progression through the molt. Once this initial ETH release reached the threshold necessary to induce EH release, the mutually stimulatory relationship between EH and ETH would then ensure the phasic and complete release of ETH.

\section{Role of EH and ETH in tracheal air filling}

Larvae genetically deficient for ETH show severely delayed respiratory dynamics, and these defects are rescued by exogenous
ETH, suggesting that the ETH peptides control this physiological response (Park et al., 2002). However, EH KO larvae (McNabb et al., 1997; this study) and pharate adults (Baker et al., 1999) also show defects in tracheal air filling. In addition, injection of $8-\mathrm{Br}$ cGMP into either wild-type or EH KO larvae caused the premature air filling of the trachea, which was normal in terms of its overall duration, completeness, and coordination among the two main tracheal trunks of the larva. Such injections also rescued the defects in respiratory dynamics expressed at adult eclosion by $\mathrm{EH}$ KO flies (Baker et al., 1999). In contrast, we found that ETH injections were less effective in inducing normal air filling in $\mathrm{EH}$ KO larvae, even at the highest doses tested. Overall, these findings suggest that air filling is mostly caused by EH, implying that the failure of ETH mutants to fill their trachea normally is indirect, and is attributable to a lack of EH release in these mutants. Examining the extent of EH release in ETH mutants would provide support for this hypothesis.

Although ETH injections did not rescue normal tracheal dynamics in EH KO larvae, they did cause some air filling to occur. In addition, although tracheal air filling was severely disrupted in EH KO larvae, most animals succeeded in filling their trachea at least partially by the onset of ecdysis. These results point to a dual control of tracheal air filling, with EH and ETH playing major and minor roles, respectively (Fig. 8, [4]). Injections of synthetic $\mathrm{EH}$ would aid in the disentangling of direct versus indirect ETH and $\mathrm{EH}$ actions. Unfortunately, synthetic Drosophila EH was not available, and synthetic Manduca EH (Eldridge et al., 1991) caused severe behavioral abnormalities and fatal physiological changes when tested on fly larvae (M. L. del Campo and J. Ewer, unpublished observation), so its use was not pursued further.

\section{Degeneracy in the control of larval ecdysis by neuropeptides}

We found that EH+CCAP KO larvae expressed much more severe defects at ecdysis than did $\mathrm{EH} \mathrm{KO}$ larvae, suggesting that $\mathrm{EH}$ and CCAP may play partially redundant roles (Fig. 8, [8]). This is a novel finding because, until now, $\mathrm{EH}$ has been considered to act purely "upstream" of CCAP (Fig. 8, [7]) (for review, see Ewer and Reynolds, 2002). Nevertheless, such degeneracy (cf. Edelman and Gally, 2001; Greenspan, 2001) would explain why CCAP KO (Park et al., 2003) and EH KO larvae are able to express ecdysis behavior. Indeed, CCAP KO larvae release $\mathrm{EH}$ (as suggested by the cGMP increases seen in Inka cells at pre-ecdysis) (Fig. 6E), and $\mathrm{EH} \mathrm{KO}$ larvae do release CCAP, although the extent of this release is not as complete as is seen in wild-type larvae (Clark and Ewer, unpublished observation). Whether this degeneracy is mediated by the EH and CCAP peptides themselves or by other factors present in these neurons will have to wait until mutants of the $\mathrm{EH}$ and CCAP genes become available.

\section{Role of EH and ETH in pre-ecdysis}

Several independent lines of evidence suggest that ETH causes pre-ecdysis "directly" and that the ability of EH to induce preecdysis is indirect and occurs because EH causes ETH release (Zitnan et al., 1996; Park et al., 2002) (for review, see Ewer and Reynolds, 2002). The inability of exogenous ETH to cause premature pre-ecdysis and ecdysis in EH KO larvae is, in light of this evidence, particularly puzzling. Such insensitivity has been noted previously but using Manduca ETH (McNabb et al., 1997; Baker et al., 1999), raising the possibility that it was attributable to differences between the moth and the fly peptides. Here, we confirmed this result using the Drosophila peptides. Thus, it appears that the EH neurons are required in Drosophila for exogenous ETH to cause premature pre-ecdysis and ecdysis (Fig. 8, [6]), 
independent of their role in causing normal ETH release from endogenous stores (Fig. 8, [3]). How can this result be reconciled with the obligatory role of ETH in the control of pre-ecdysis and ecdysis inferred from the phenotype of ETH mutants (Park et al., 2002)? One possibility is that additional ecdysiotropic neuropeptides exist that would also be released in response to EH. These neuropeptides would presumably be expressed and released within the CNS, because ETH can elicit pre-ecdysis in an isolated Manduca CNS (Zitnan et al., 1996, 1999). In this scenario, larvae lacking ETH would not express pre-ecdysis because of the failure to cause the $\mathrm{EH}$ release, which in turn induces the secretion of these additional peptides. Likewise, injection of ETH into EH KO larvae would also be without effect, because $\mathrm{EH}$ release would never be induced. Although this hypothesis can accommodate the results discussed above, it is difficult to reconcile with the fact that EH KO larvae express pre-ecdysis and ecdysis behaviors and, furthermore, express these behaviors on a normal (albeit ETH insensitive) schedule. Alternatively or additionally, EH may be one of several factors that can regulate ETH signaling via mechanisms at or downstream of ETH receptors (Fig. 8, [6]). Clearly, this question merits additional investigation, especially because other Drosophila neuropeptides exist that can cause ecdysis [e.g., the products of the huggin gene (Meng et al., 2002)].

Ecdysis is a stereotyped behavior that is essential to the survival of all insects. The virtually invariant and flawless deployment of the complex physiological and behavioral events that are expressed at this time is attributable to the intricate coordination and actions of several neuropeptides (Fig. 8). Here we identified additional complexities in the control of this behavior that provide general insights into how neuropeptides trigger changes in behavior and physiology at the appropriate time and in the correct temporal sequence.

\section{References}

Baker JD, McNabb SL, Truman JW (1999) The hormonal coordination of behavior and physiology at adult ecdysis in Drosophila melanogaster. J Exp Biol 202:3037-3048.

Edelman GM, Gally JA (2001) Degeneracy and complexity in biological systems. Proc Natl Acad Sci USA 98:13763-13768.

Eldridge R, Horodyski FM, Morton DB, O'Reilly DR, Truman JW, Riddiford LM, Miller LK (1991) Expression of an eclosion hormone gene in insect cells using baculovirus vectors. Insect Biochem 21:341-351.

Ewer J, Reynolds S (2002) Neuropeptide control of molting in insects. In: Hormones, brain and behavior (Pfaff DW, Arnold AP, Fahrbach SE, Etgen AM, Rubin RT, eds), pp 1-92. San Diego: Academic.

Ewer J, Truman JW (1996) Increases in cyclic 3', 5' -guanosine monophosphate (cGMP) occur at ecdysis in an evolutionarily conserved crustacean cardioactive peptide-immunoreactive insect neuronal network. J Comp Neurol 370:330-341.

Ewer J, Gammie SC, Truman JW (1997) Control of insect ecdysis by a positive-feedback endocrine system: roles of eclosion hormone and ecdysis triggering hormone. J Exp Biol 200:869-881.

Gammie SC, Truman JW (1997) Neuropeptide hierarchies and the activation of sequential motor behaviors in the hawkmoth, Manduca sexta. J Neurosci 17:4389-4397.

Greenspan RJ (2001) The flexible genome. Nat Rev Genet 2:383-387.

Horodyski FM, Ewer J, Riddiford LM, Truman JW (1993) Isolation, char- acterization and expression of the eclosion hormone gene of Drosophila melanogaster. Eur J Biochem 215:221-228.

Husain QM, Ewer J (2004) Use of targetable gfp-tagged neuropeptide for visualizing neuropeptide release following execution of a behavior. J Neurobiol 59:181-191.

Kingan TG, Adams ME (2000) Ecdysteroids regulate secretory competence in inka cells. J Exp Biol 203:3011-3018.

Kingan TG, Gray W, Zitnan D, Adams ME (1997) Regulation of ecdysistriggering hormone release by eclosion hormone. J Exp Biol 200:3245-3256.

Kingan TG, Cardullo RA, Adams ME (2001) Signal transduction in eclosion hormone-induced secretion of ecdysis-triggering hormone. J Biol Chem 276:25136-25142.

McNabb SL, Baker JD, Agapite J, Steller H, Riddiford LM, Truman JW (1997) Disruption of behavioral sequence by targeted death of peptidergic neurons in Drosophila. Neuron 19:813-823.

Meng X, Wahlstrom G, Immonen T, Kolmer M, Tirronen M, Predel R, Kalkkinen N, Heino T, Sariola H, Roos C (2002) The Drosophila hugin gene codes for myostimulatory and ecdysis-modifying neuropeptides. Mech Dev 117:5-13.

Miller MW, AA, Cropper EC, Vilim FS, Karagogeos D, Kupfermann I, Weiss KR (1991) Localization of myomodulin-like immunoreactivity in the central nervous system and peripheral tissues of Aplysia californica. J Comp Neurol 314:627-644.

Nässel DR (2000) Functional roles of neuropeptides in the insect central nervous system. Naturwissenschaften 87:439-449.

Nässel DR (2002) Neuropeptides in the nervous system of Drosophila and other insects: multiple roles as neuromodulators and neurohormones. Prog Neurobiol 68:1-84.

O'Brien MA, Taghert PH (1998) A peritracheal neuropeptide system in insects: release of myomodulin-like peptides at ecdysis. J Exp Biol 201:193-209.

Park J, Schroeder AJ, Helfrich-Förster C, Jackson FR, Ewer J (2003) Targeted ablation of CCAP neuropeptide-containing neurons of Drosophila causes specific defects in execution and circadian timing of behavior. Development 130:2645-2656.

Park Y, Zitnan D, Gill SS, Adams ME (1999) Molecular cloning and biological activity of ecdysis-triggering hormones in Drosophila melanogaster. FEBS Lett 463:133-138.

Park Y, Filippov V, Gill SS, Adams ME (2002) Deletion of the ecdysistriggering hormone gene leads to a lethal ecdysis deficiency. Development 129:493-503.

Predel R, Eckert M (2000) Neurosecretion: peptidergic systems in insects. Naturwissenschaften 87:343-350.

Strand FL (1999) Neuropeptides: regulators of physiological processes. Cambridge, MA: MIT.

Truman JW, Mumby SM, Welch SK (1979) Involvement of cyclic GMP in the release of stereotyped behavior patterns in moths by a peptide hormone. J Exp Biol 84:201-212.

Zitnan D, Kingan TG, Hermesman JL, Adams ME (1996) Identification of ecdysis-triggering hormone from epitracheal endocrine system. Science 271:88-91.

Zitnan D, Ross LS, Zitnanova I, Hermesman JL, Gill SS, Adams ME (1999) Steroid induction of a peptide hormone gene leads to orchestration of a defined behavioral sequence. Neuron 23:523-535.

Zitnan D, Hollar L, Spalovska II, Takac P, Zitnanova II, Gill SS, Adams ME (2002) Molecular cloning and function of ecdysis-triggering hormones in the silkworm Bombyx mori. J Exp Biol 205:3459-3473.

Zitnan D, Zitnanova I, Spalovska II, Takac P, Park Y, Adams ME (2003) Conservation of ecdysis-triggering hormone signaling in insects. J Exp Biol 206:1275-1289. 